\title{
A Stackelberg Game for Spectrum Investment and Pricing in Cooperative Cognitive Radio Networks
}

\author{
Heejun Roh, Hoorin Park, Cheoulhoon Jung and Wonjun Lee \\ Department of Computer Science and Engineering \\ WCU Future Network Optimization Technology Center (FNOT) \\ Korea University, Seoul, Republic of Korea \\ wlee@korea.ac.kr
}

\begin{abstract}
Recently, a new paradigm named cooperative cognitive radio networks (CCRNs) is proposed by many researchers to realize cooperative communication and cognitive radio technologies. In CCRN consisting of primary users (PUs), secondary users (SUs), and the secondary AP (SAP), the leasing and pricing of spectrum bands and cooperative spectrum sensing is occurred and SUs relay packets to get the temporal spectrum permission. These behaviors can be modeled by using multi-stage Stackelberg game, and some previous results are already available. However, since existing CCRN studies did not consider spectrum pricing, we propose a new CCRN model which SUs' cooperation is coordinated by SAP to reduce leasing cost, based on a simple multi-stage Stackelberg game. Our preliminary result shows some distinct characteristics of CCRN which affect the optimal strategy.
\end{abstract}

\section{Categories and Subject Descriptors}

C.2.1 [Computer-Communication Networks]: Network Architecture and Design-Wireless Communication

\section{General Terms}

Algorithm, Design, Economics

\section{INTRODUCTION}

With the advance of physical communication technologies, many wireless communication technologies are suggested to use wireless resources efficiently. Amongst such technologies, cooperative communication (CC) and

Permission to make digital or hard copies of all or part of this work for personal or classroom use is granted without fee provided that copies are not made or distributed for profit or commercial advantage and that copies bear this notice and the full citation on the first page. To copy otherwise, to republish, to post on servers or to redistribute to lists, requires prior specific permission and/or a fee.

ACM CoNEXT Student Workshop, December 6, 2011, Tokyo, Japan.

Copyright 2011 ACM 978-1-4503-1042-0/11/0012 _...\$10.00. cognitive radio (CR) are promising technologies for future network design. CC is based on the collaboration among neighbor users and leads improved connectivity and throughput in opportunistic manner. CR enables efficient and flexible spectrum usage by technological cognition of spectrum hole.

Many researchers suggest a combined network model named cooperative cognitive radio networks (CCRN). [3], [4] Like CR, typical CCRN classifies users as primary users (PUs) and secondary users (SUs). PU has rights to use spectrum bands, but SU cannot use the bands without PUs' permission. In CCRN, SUs cooperate a PU to get some redundant, time-temporal bandwidth by relaying packets. Sometimes, a SU can pay the permission fees to a $\mathrm{PU}$ in some situations.

On the other hand, recent CRN studies suggest the existence of spectrum brokers or secondary AP (SAP) to describe the interface between the traditional spectrum licensees (PUs) and the SUs. A spectrum broker leases part of the unused spectrum from PUs, senses the active bands used by PUs for spectrum holes, or resells the bandwidth to the SUs to maximize profit. [1]

In this paper, we formulate and observe a multi-stage Stackelberg game for understanding spectrum investment and pricing in CCRNs. Existing game theoretical approaches do not consider the impact of cooperation [1], or multiple spectrum bands [4], [3]. Especially, existing CCRN models typically assume TDMAbased spectrum allocation algorithms and do not consider spectrum holes, which are not realistic assumptions. Our CCRN model solves the above problems by analyzing the impact of cooperation, spectrum leasing, and pricing at the same time. Note that our model on cooperation and spectrum leasing can be applied to the infrastructure-based networks.

\section{STACKELBERG GAME ANALYSIS}

Our 4-stage Stackelberg game for CCRNs is as follows. In our game, SAP is the leader and the set of SUs $S=\{1,2, \ldots, n\}$ is the follower. In stage I, SAP determines the probability $p$ that each $\mathrm{SU}$ cooperates in the 
overlay band. After stage I, $p$ is announced to SUs and $\lfloor n p\rfloor$ SUs try to do cooperation with PUs. According to the spatio-temporality of spectrum band usage, SUs report the available bandwidth $\alpha\lfloor n p\rfloor W$ can be obtained, where $W$ is the maximum achievable bandwidth of a SU in the overlay band and $0 \leq \alpha \leq 1$. Note that right to use the available bandwidth is given to SAP by paying cooperation cost. In stage II, SAP determines leasing amount $W_{l}$ based on the available bandwidth $\alpha\lfloor n p\rfloor W$. In stage III, SAP announces the price $\pi$ to the SUs. Finally, SUs choose their bandwidth demands to maximize their individual payoff function in stage IV. Note that this multi-stage Stackelberg game can be analyzed using the backward induction algorithm [2]. Due to the limited space, we only include the basic equations to explain the above game model in this paper.

\subsection{SUs' Optimal Spectrum Demand Strategy}

In our model, user $i$ 's achievable rate can be described as $r(i)=w_{i} \log \left(1+\frac{g_{i}}{w_{i}}\right)$, where $w_{i}$ is the allocated bandwidth, $g_{i}$ is the band gain. To simplify our analysis, we assume that $r(i) \approx w_{i} \log \left(\frac{g_{i}}{w_{i}}\right)$ as in [1]. Then user $i$ 's utility function can be described as the difference of its achievable rate and payment for band $w_{i}: u_{i}\left(\pi, w_{i}\right)=w_{i} \log \left(\frac{g_{i}}{w_{i}}\right)-\pi w_{i}$, which implies that the optimal spectrum demand is achieved as $w_{i}^{*}(\pi)=\arg \max _{w_{i} \geq 0} u_{i}\left(\pi, w_{i}\right)=g_{i} \exp (-(1+\pi))$. Therefore, the total users' demand is $\sum_{i=1}^{n} w_{i}^{*}(\pi)=$ $G \exp (-(1+\pi))$, where $G=\sum_{i=1}^{n} g_{i}$. This optimal SUs' demand strategy is applied in Stage IV.

\subsection{SAP's Optimal Spectrum Investment and Pricing}

In our model, SAP must pay for two kinds of costs: leasing cost and cooperation cost, since SAP leases $W_{l}$ units of bandwidth from spectrum owner and uses SUs' cooperation capability by announcing $p$. We denote the unit leasing cost and the unit cooperation cost to $C_{l}$ and $C_{c}$, respectively. Then the SAP's profit is

$$
\begin{aligned}
R\left(p, \alpha, W_{l}, \pi\right)= & \pi \min \left(G e^{-(1+\pi)}, W_{l}+\alpha\lfloor n p\rfloor W\right) \\
& -\alpha\lfloor n p\rfloor W C_{c}-W_{l} C_{l} .
\end{aligned}
$$

Therefore, in Stage III, SAP announces the optimal price $\pi^{*}=\arg \max _{\pi>0}=R\left(p, \alpha, W_{l}, \pi\right)$, and in Stage II, SAP determines the optimal leasing amount $W_{l}^{*}=$ $\arg \max _{W_{l}} R\left(p, \alpha, W_{l}, \pi^{*}\right)$. Finally, in Stage I, given $f_{\alpha}(x)$, the probability distribution function of $\alpha$, SAP determines the optimal cooperation probability $p^{*}=$ $\arg \max _{0 \leq p \leq 1} \int_{0}^{1} R\left(p, \alpha, W_{l}^{*}, \pi^{*}\right) f_{\alpha}(x) d x$. If $n$ and the distribution of $\alpha$ are known, the optimal strategy can be achieved numerically. According to our simplified derivation, the relationship between demand-price product and supply-price product in stage III, cooperation revenue (achievable bandwidth) in stage II, and the relationship between cooperation cost and leasing cost determine the optimal strategies.

\subsection{Observations}

At a glance, our Stackelberg game for CCRN is similar to the Stackelberg game for CRN in [1]. However, there are different characteristics which need to discuss more deeply including:

- Since some SUs increase spectrum resource production in the spectrum market by cooperation, the number of SUs $n$ has more impact on pricing and investment.

- In [1], it is assumed that the realization factor $\alpha$ is uniformly distributed. However, CCRN needs more complicated probabilistic model for $\alpha$, since modeling PU's traffic affects $\alpha$ considerably.

\section{CONCLUSION AND FUTURE WORK}

In this paper, a simple Stackelberg game for CCRN to analyze the relationship between investment (i.e., cooperation and leasing) and pricing in infrastructure-based CCRNs is suggested. Our contribution is mainly on the formulation of Stackelberg game and observations with several assumptions to understand distinct characteristics of CCRN. Our future work focuses on the relaxation of such assumptions and detailed modeling of PU traffic.

\section{ACKNOWLEDGMENTS}

This research was jointly sponsored by MEST, Korea under WCU (R33-2008-000-10044-0), MKE, Korea under ITRC NIPA-2011-(C1090-1121-0008), MEST, Korea under Basic Science Research Program (2011-0012216), and NRF of Korea under the project of Global Ph.D. Fellowship conducted from 2011. Wonjun Lee is the corresponding author.

\section{REFERENCES}

[1] L. Duan, J. Huang, and B. Shou. Cognitive mobile virtual network operator: Investment and pricing with supply uncertainty. In Proceedings of IEEE INFOCOM, pages 1-9, March 2010.

[2] G. A. Jehle and P. J. Reny. Advanced Microeconomic Theory. Prentice Hall, third edition, 2011.

[3] Y. Yi, J. Zhang, Q. Zhang, T. Jiang, and J. Zhang. Cooperative communication-aware spectrum leasing in cognitive radio networks. In Proceedings of IEEE DySPAN, pages 1-11, April 2010.

[4] J. Zhang and Q. Zhang. Stackelberg game for utility-based cooperative cognitive radio networks. In Proceedings of ACM MobiHoc, pages 23-32, May 2009. 